\title{
MÁS ALLÁ... DE LA NIC-38 \\ [Una mirada al futuro de los Activos Intangibles]
}

\author{
BEYOND IAS 38
}

[A look to the future of Intangible Assets]

[A look to the future of Intangible Assets]
ARTURo Roman Valdivia RaMos*
Docente Auxiliar de la Facultad de Ciencias Contables
Universidad Nacional Mayor de San Marcos - UNMSM
Lima-Perú
[Recepción: Setiembre de 2012 / Conformidad: Octubre de 2012]

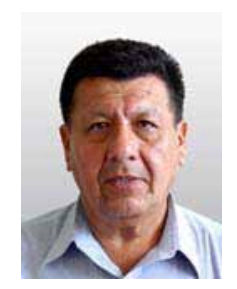

\section{RESUMEN}

Los Activos Intangibles, son activos que generan y crean valor a las cosas. El producto por sí mismo no crea valor, el valor se lo da la marca, la reputación, la cultura corporativa ...

Por lo tanto, es difícil que la Contabilidad actual pueda registrar estos Activos Intangibles, pues no fue creada y diseñada para ello. La Contabilidad, está hecha para registrar cosas tangibles o materiales, dándole un valor económico a tales cosas.

¿Vendería su empresa por la cantidad que refleja la Contabilidad?

Seguro que la respuesta será NO, ya que existe número indeterminado de intangibles que elevarán el precio de venta, es decir lo que llamamos valor extra-contable.

Sin embargo, la Contabilidad registra algunos activos intangibles, pero estos son pocos:

1. Concesiones

2. Ciencias y otros Derechos

3. Patentes

4. Propiedad Industrial (Marcas)

5. Programas de Computadora (Software)

6. Costos de explotación y desarrollo

7. Fórmulas, diseños y prototipos

8. Reservas de Recursos Extraíbles

9. Plusvalía Mercantil

10. Otros Activos Intangibles

$\mathrm{Y}$ terminamos de contar. Entonces, la actual Contabilidad a través de la NIC-38 Activos Intangibles, solo puede medir y contabilizar estos intangibles a excepción del numeral 10. ¿Pero... cuales son los Otros Activos Intangibles?

Palabras clave: Activos Intangibles, instrumentos no financieros de medición, valor agregado, NIC-38, prospectiva estratégica.

\section{ABSTRACT}

Intangible assets are assets that generate and create value things. The product itself does not create value; the value is what gives the brand, reputation, corporate culture.

Therefore, it is difficult that the current accounting can record these intangible assets, as it was not created and designed for it. Accounting is made to record tangible or material things, giving an economic value to such things.

Would you sell your company for the amount that reflects the accounting?

Surely the answer is NO, because there is any number of intangibles that will raise the sales price, that is what we call extra-value accounting.

However, the accounting records some intangible assets, and these are a few:

1. Concessions

2. Science and Other Rights

3. Patents

4. Industrial Property (Brands)

5. Computer Programs (Software)

6. Development and operational costs

7. Formulas, designs and prototypes

8. Reservations of Extractable Resource

9. Goodwill

10. Other Intangible Assets

And we finished counting. Then, the current Accounting through the NIC-38 Intangible Assets, only can measure and account these intangibles except the numeral 10.

But ... what are the other Intangible Assets?

Keywords: Intangible Assets, non-financial instruments measurement, added value, IAS38 , strategic foresight.

\footnotetext{
* Doctorante en Ingeniería Industrial. Docente investigador-UNMSM. E-mail: arturo.valdivia.ramos@gmail.com
} 


\section{EL MUNDO ES DE LOS INTANGIBLES}

Si tuviéramos una balanza enorme, gigantesca, donde en un plato colocáramos los tangibles o materiales y en el otro plato de la balanza, colocáramos los intangibles o inmateriales, de seguro que este último se inclinaría a su favor. Y es que en toda empresa, incluso de producción, industrial o manufactura, predominan los intangibles y siempre ha sido así; lo que ha sucedido es que al no ver visto o tocado, no ha podido ser Contabilizado, pero sin medido.

Desde luego esta medición es cualitativa y la Contabilidad requiere que sea cuantitativa para reflejarla en dinero.

En EE.UU hace 88 años (1924), se realizó un estudio para conocer y/o determinar el valor de la empresa y los resultados que dicho estudio arrojó, fue el siguiente:

\begin{tabular}{|c|c|c|c|}
\hline PAIS & ANo & $\begin{array}{c}\text { VALOR } \\
\text { TANGIBLE }\end{array}$ & $\begin{array}{c}\text { VALOR } \\
\text { INTANGIBLE }\end{array}$ \\
\hline USA & 1924 & $75 \%$ & $25 \%$ \\
\hline
\end{tabular}

Pero, en el año 2012, las cosas son totalmente diferentes. Veamos lo que sucedió con el transcurrir de los años:

\begin{tabular}{|l|l|l|l|}
\hline USA & 1980 & $25 \%$ & $75 \%$ \\
\hline USA & 1990 & $20 \%$ & $80 \%$ \\
\hline USA & 2000 & $10 \%$ & $90 \%$ \\
\hline USA & 2005 & $5 \%$ & $95 \%$ \\
\hline USA & 2010 & $1 \%$ & $99 \%$ \\
\hline
\end{tabular}

Y para no irnos a un país lejano, veamos lo que sucede en el Brasil que es un país amigo y vecino nuestro.

BRASIL

Valor de Mercado de las Empresas de Capital Abierto

\begin{tabular}{|c|c|c|}
\hline AÑO & INTANGIBLE & TANGIBLE \\
\hline 1981 & $17 \%$ & $86 \%$ \\
\hline 1998 & $71 \%$ & $29 \%$ \\
\hline 2005 & $80 \%$ & $20 \%$ \\
\hline 2010 & $90 \%$ & $10 \%$ \\
\hline
\end{tabular}

Como podemos apreciar, los activos intangibles a medida que van pasando los años, va creciendo en valor e importancia.

$¿ Y$ hasta donde vamos a llegar?

¿Acaso, en el futuro, los tangibles ya no tendrán impacto en el valor de la empresa?

Y si esto, fuese así, entonces que estaría pasando con la Contabilidad actual.

¿Estaría la Contabilidad demostrando el Patrimonio en forma real y verídica?

¡Este es el reto actual que tiene la Contabilidad!

\section{OTROS ACTIVOS INTANGIBLES}

Las empresas e instituciones están llenas de intangibles y aquellos que consideran que no los tienen o lo tienen poco, están grandemente equivocados. Todo está lleno de intangibles o inmateriales. Es una oficina, sin importar lo que haga, solo tiene unos cuantos escritorios, sillas, anaqueles, estantes y archivadores; además de unos cuadros, una mesa de centro y útiles de escritorio y de oficina.

Pero estos son pocos en comparación con todos los intangibles o inmateriales que existen y están presentes en la misma oficina, como son, el oxígeno, la colocación de los bienes, la ergonomía, la vista hacia afuera, la luminosidad, el confort, el aseo, la limpieza, los colores, el ruido, y cientos de intangibles, más. Por cada tangible que hay en una oficina, ambiente de trabajo o taller, hay 10 intangibles (como promedio), que existen en el mismo lugar, por lo tanto la relación entre tangible e intangible es grande, a favor del último.

Es el cuerpo humano, la relación aún es mayor a favor de los intangibles, pues el cuerpo humano está compuesto de órganos, músculos, nervios, estructura ósea, entre otros; pero más tiene intangibles como el pensamiento, actitud, valores, ética, incluso 
sueños. Siempre entonces, los intangibles superarán a los tangibles.

En resumen, la Contabilidad tiene una pequeña o limitada capacidad de registrar activos intangibles.

\section{ALGUNAS DEFINICIONES DE ACTIVO INTAGIBLE}

\section{1. ¿Qué es un Activo Intangible?}

... si bien la mayoría de las definiciones parecen estar de acuerdo en que los Activos Intangibles son origen probable de beneficios económicos futuros, que carecen de sustancia física, que son controlados por la firma como resultado de eventos anteriores o transacciones... todavía existe la necesidad de una definición generalmente aceptada.

$$
\begin{array}{r}
\text { Cañibano García - Ayuso y Sánchez } \\
1999
\end{array}
$$

2. Un Activo Intangible es un activo identificable, de carácter no monetario y sin apariencia física.

$$
\text { NIC-38 (Activos Intangibles) }
$$

Enero 2012

3. Debemos entender por activos intangibles como aquellos activos no monetarios identificables (activos capaces de generar beneficios económicos), sin sustancia física, que generarán beneficios económicos futuros controlados por la empresa.

$$
\text { Tania Delgado Martínez }
$$

\section{Activo Intangible}

Un bien no físico, que a medida que pasa el tiempo (se acerca al futuro), aumenta de valor.

Arturo Valdivia Ramos

2012
Se puede apreciar que la definición de Activo Intangible es más corta o abreviada a medida que pasa el tiempo. $Y$ debe ser así, hasta encontrar una definición exacta y precisa. Como dice Cañibano, todavía existe la necesidad de una definición generalmente aceptada.

\section{CUÁLES SERÍAN LOS OTROS ACTI- VOS INTANGIBLES}

Como hemos mencionado, existen más intangibles que tangibles en una organización. En una empresa, tenemos materias primas, insumos, materiales, partes, piezas, productos, maquinarias y equipos, infraestructura, dinero y termino de contar.

En cambio tenemos: estructuras organizacionales, clientes, ergonomía, satisfacción del cliente, deseo, esperanza, aspiraciones, know-how, desempeño, rendimiento, reputación, servicio, bienestar, felicidad, ambiente laboral, cultura corporativa, medio - ambiente, incluso oxígeno que respiramos son intangibles, pero que la Contabilidad actual aún no está preparada para registrar contablemente estos activos intangibles, que dan valor agregado a la empresa. Al igual que cambia la economía o la administración, la Contabilidad debe cambiar también. Las Normas Contables que eran válidas para el pasado, no lo son la actualidad para los activos intangibles y con esos instrumentos $y$ normativas queremos medir y contabilizar dichos activos. Es por eso, que son muchas las inversiones en intangibles que no satisfacen los requisitos de reconocimiento contable, y ello trae como consecuencia, que no puedan ser identificados en los Balances y EE.FF de las empresas. 


\section{LOS ACTIVO INTAGIBLES EN EL TIEMPO}

Los Activo Intangibles en el futuro, tendrán más valor de los que tienen en la actualidad. A medida que se va incrementando el tiempo, mayor valor tendrá; es decir, es al revés de lo que sucede con los productos o mercancías, a medida que pasa el tiempo, estos se deterioran, se gasta o consumen y con el paso del tiempo, pierden de valor.

Hoy en días, tener una formidable infraestructura o una maquinaria de punta, no garantiza a la empresa una ventaja competitiva. $Y$ esto es un Activo Tangible o material.

Sin embargo, más importante es la innovación, clientes leales a nuestro producto $y / o$ servicio, la credibilidad de su personal directivo, etc. El tiempo, es el mejor aliado del activo intangible y el espacio, lugar preciso por donde recorre este material. Debemos por lo tanto definir, medir, valorar, controlar y gestionar el activo intangible, ya que es el aspecto fundamental para la competitividad empresarial dentro del actual contexto socioeconómico, en que nos encontramos inmersos.

Es decir, para ser exitosas las empresas, estas no se pueden centrar exclusivamente en fabricar o producir bienes o productos, sino que debe invertir en activos intangibles que son los que generan valores futuros.

\section{NUEVOS INSTRUMENTOS NO FINANCIEROS PARA MEDIR ACTIVOS INTANGIBLES}

Los Activos Intangibles vienen a ser la diferencia que existe entre el resultado contable reflejado en los EE.FF y el valor real de la empresa en el mercado o que puede tener para sus socios o accionistas. Estos Activos Intangibles como la Marca, lema comercial, prestigio, años de presencia en el mercado, clientes cautivos, entre otros; son los que le dan mayor valor a la empresa, incluso mucho más que una impresionante infraestructura y máquinas o equipos de última generación, generación que pueden ser muy costosos.

Sin embargo, los activos Intangibles son difíciles de medir, cuantificar o valorar y desde luego muy complejo poder contabilizarlos y transferirlo en valores monetarios. Creo sinceramente que con la actual normatividad contable existente, es difícil poder contabilizados, tendríamos que crear, diseñar, implementar o simplemente utilizar otros instrumentos aún poco conocidos por la ciencia contable, pero sí de utilización en otras áreas empresariales; por lo tanto, la tarea de contabilizar los nuevos Activos Intangibles es y será una tarea multi-disciplinaria, donde intervendrán y actuarán, psicólogos, sociólogos, antropólogos, economistas, administradores, ingenieros, abogados, entre otros profesionales, pero todos bajo la conducción y gestión del contador, pues es el responsable de su contabilización.

\section{COMO CONOCER EL VALOR AGREGADO POR INTANGIBLES}

Nada mejor que un Ejemplo-Práctico para comprender el valor agregado que da el Intangible a un producto $\mathrm{y} / \mathrm{o}$ servicio.

\begin{tabular}{|c|c|}
\hline & POLLO A LA BRASA \\
\hline \multirow[b]{2}{*}{ PRODUCTO } & $\begin{array}{l}\text { Materia Prima } \\
\text { Pollo }(1.500 \mathrm{~kg}): 8.20 \text { soles }\end{array}$ \\
\hline & $\begin{array}{l}\text { Producto } \\
\text { Pollo a la brasa: } 2.30 \text { soles }\end{array}$ \\
\hline \multirow{5}{*}{ SERVICIO } & $\begin{array}{l}\text { EL INTANGIBLE CAMBIO EL VALOR AL } \\
\text { PRODUCTO }\end{array}$ \\
\hline & $\begin{array}{l}\text { Marca } \\
\text { Pardo's Chiken : } 27.80 \text { soles }\end{array}$ \\
\hline & $\begin{array}{l}\text { Servicios } \\
\text { Pardo's Chiken : } 1.40 \text { soles }\end{array}$ \\
\hline & $\begin{array}{l}\text { Experiencia } \\
\text { Pardo's Chiken : } 4.30 \text { soles }\end{array}$ \\
\hline & Total: $\quad 44.00$ soles \\
\hline
\end{tabular}


Como se puede apreciar el Intangible Marca, Servicio y Experiencia, es lo que le da valor agregado al producto en este caso al pollo a la brasa. Mientras más prestigio tenga la Marca, más valor le agregará al producto que comercializa o venda. Y así, sucede con todos los productos. No existe ningún producto que no tenga un intangible que lo rodee, así sea arena o piedra, pues su valor lo dará la cantera o lugar de donde ha sido extraído.

No vale igual la madera que extraemos de un bosque natural, que la que extraemos de un bosque natural protegido por el estado, ni es igual que este haya sido talado por mujeres y niños que por hombres.

Entonces, podemos deducir que a cualquier producto, se le puede y debe agregar intangible(s) para incrementar se valor. Un intangible, debe tener en cuenta el tiempo, que es el único recurso que no es renovable. Todo cambia rápidamente a nuestro alrededor.

El valor ya no lo da el producto (y la Contabilidad solo registra tangibles y algunos servicios), sino que son los intangibles la frente de todo valor. Como resultado de ello, la capacidad de información de los EE.FF sobre la situación financiera de la empresa no es tan confiable o por decir lo menos, su veracidad está disminuyendo considerablemente.

En un futuro cercano, los activos intangibles que si se pueden medir a través de porcentaje, peso, volumen o masa, se deberán colocar en los EE.FF de una compañía.

¡Cómo no ha de tener valor económico la marca, la atención al cliente, la reputación de la empresa, la calidad del servicio! Al respecto, Rómulo Mesarina de Zela, ha dicho: "la calidad de un producto no necesariamente está en el producto mismo.

Por el contrario, la calidad está en la percepción del consumidor y es hacia allá adonde debe apuntarse si se busca crear una marca fuerte, que es un intangible con un gran valor".

\section{EL ANÁLISIS CONTABLE ES POCO ADECUADO PARA VALORIZAR LOS OTROS ACTIVOS INTANGIBLES}

Con la actual Contabilidad que conocemos, no es posible registrar los Otros Activos Intangibles. La Contabilidad fue creada y hecha (como lo hemos mencionado anteriormente), para contabilizar productos, insumos, materias primas, materiales, partes, piezas, máquinas y equipos, infraestructura, muebles, inmuebles, valores, dinero y cientos de objetos o mercancías que son materiales o tangibles. Los creadores de la Contabilidad, ni siquiera pensaron que su creación iba más allá: los intangibles, pues en aquellos tiempos, nadie se podía haber imaginado cosas que actualmente son más importantes que los productos o mercancías.

Entonces, si la Contabilidad aún no puede registrar contablemente los intangibles, entonces la información que proporciona, no es del todo completa.

En otras palabras, para la Contabilidad le resulta difícil Contabilizar los intangibles, pues este se guía por principios tradicionales que valoriza sólo lo que es razonablemente mensurable o cuantitativo y esto lo refleja en sus EE.FF, pero no registra los factores relevantes como son los activos intangibles de una empresa.

Por otro lado, dejar de utilizar los actuales sistemas contables para registrar los intangibles, no es aconsejable ni en el corto y mediano plazo, ya que conllevaría un costo enorme, por lo tanto, parece ser que el procedimiento más sensato para incrementar mayos veracidad a los EE.FF., será la de ir desarrollando instrumentos-herramientas que sirvan de complemento al marco del sistema contable que hoy tenemos.

De esta forma entonces podremos decir, que los EE.FF, que incluyan o anexen valoraciones de activos intangibles es fiel imagen del Patrimonio de la empresa. 
También debemos tener presente que otro problema de la Contabilidad es determinar realmente que son los intangibles y como tratarlos como un activo, a pesar de lo que señala la SFACS (FASB)

\section{ALGUNOS INSTRUMENTOS - HERRAMNIENTAS DE MEDICIÓN DE INTANGIBLES}

En la actualidad, existen diversos Instrumentos - Herramientas de medición de intangibles, que nos permiten obtener un resultado cualitativo o cuantitativo pero sin llegar a valores monetarios.

Leif Edvinsson, señaló lo siguiente: "Una corporación es como un árbol. Hay una parte que es visible (las frutas) y una parte que es oculta (las raíces), y si solamente te preocupas por las frutas, el árbol puede morir. Para que el árbol crezca y continúe dando frutas es necesario que las raíces estén sanas y nutridas. Esto es válido para las empresas: Si sólo nos concentramos en los resultados financieros, e ignoramos los valores escondidos (intangibles), la compañía no subsistirá en el corto plazo".

Los estudiosos e investigadores empresariales, han realizado enormes esfuerzos que han servido para conocer esos valores escondidos, habiendo creado o diseñado, los siguientes instrumentos - herramientas para la medición de intangibles:

1. Balanced Business Scorecard

2. Intangible Assets Monitor

3. Modelo de la Universidad West Ontario

4. Modelo de Skandia Navigator

5. Technology Broker

6. Dow Chemical

7. Modelo Intelect

8. Modelo de N. Bontis

\section{Modelo de Análisis de Riesgo}

10. Modelo de Valdivia (creado por este autor), entre otros.

Pero, también tenemos otras herramientas coadyuvantes, como:

1. Medición de la Calidad del Servicio

2. Medición de la Rentabilidad del Cliente

3. Medición de la RSE/Responsabilidad Social Empresarial

4. Medición de la Preferencia de un producto

5. Medición de la Calidad del trabajo

6. Medición de la Dirección y Rentabilidad de la Empresa

7. Medición de la Gestión Empresarial

8. Medición del Rendimiento o Desempeño (Performance de una área)

9. Medición del Capital Intelectual (Guillermo Bocangel Weydert)

10. Medición de la Percepción / Satisfacción del Cliente (Voz del cliente), entre otros.

$\mathrm{Y}$ de algunas técnicas innovadoras:

1. ISO 9001:2008

2. ISO 14001:2004

3. ISO 18001

4. ISO 22000

5. Diagrama de Ishikawa / Pareto / Taguchi / Kaizen

6. Tablero de Bord

7. Cadena de Valor / Porter

8. Stakeholders

9. TOC / Theory of Constraints

10.Six sigma, entre otros.

Todos ellos sirven para medir o cuantificar intangibles y sobre todo su impacto en el cliente.

Francisco Rubira, Director de Marketing on line de TP/Páginas Amarillas de España, ha afirmado que "el futuro será cobrar por impacto" y no deja de ser verdad. 


\section{POR QUÉ ESTUDIAS LOS INTANGIBLES}

Ya se ha empezado a darle la verdadera importancia que tienen los intangibles en la empresa a través de su estudio para conocer su valor y ser considerados en los sistemas contables. Claro está, que la normalización y/o estandarización es un aspecto difícil aún de medir, valuar o cuantificar. Por otro lado, la nueva problemática que plantean las NIC y NIIF al respecto y el no establecimiento de metodologías consensuadas (aún no existen), hacen, verdaderamente difícil la contabilización de los intangibles.

Solo, cuando se logre un consenso en la aplicación del instrumento - herramienta, herramientas o técnicas antes señaladas (desde luego, pueden haber otras), recién estaremos en el camino correcto.

$Y$ es que, cuando se valora un intangible como la Marca, el Lema Comercial o simplemente el Know-How, normalmente se refleja como gasto en los registros contables, pero cuando esta misma empresa se desea vender, lo más importante y lo que atrae al posible comprador es la Marca. Entonces es una inversión. Ahí el dilema.

Tampoco pretendemos diseñar una metodología que sea consensuada o unánime, es decir, aceptada por todos (me refiero a todos los que lo comparten). Solo por citar un ejemplo, existen actualmente más de 200 formas o modelos de valorar la Marca. Esto, nos demuestra que existe una enorme preocupación por incorporar los activos intangibles a las nuevas normas contables.

Otro problema seria a enfrentar en un futuro cercano, será la gestión de estos activos intangibles. Francisco González, Presente de BBVA, ha dicho "la gestión de los intangibles, es la manera más inteligente que tienen las empresas para responder al entorno actual". Como los intangibles no tienen un reflejo cuantitativo sino cualitativo, conocer cuál es su valor económico, he ahí el problema en cuestión. Como decíamos en párrafos anteriores, esta preocupación no solo es de lo contadores, sino también de los administradores, economistas, ingenieros e incluso de sacerdotes, aunque nos perezca inverosímil.

Sabemos que el incremento del interés por los intangibles se hace cada vez más relevante, cuando se compran empresas o compañías.

Por consiguiente, el futuro de los mismos, estará en la gestión de los intangibles y para ello, la valuación o valoración es el primer paso. Y eso, estamos haciendo.

\section{NIC-38 ACTIVOS INTANGIBLES DEL FUTURO}

Como podemos apreciar, la Contabilidad trata por todos los medios a su alcance de contabilizar los activos intangibles (no los que nosotros preconizamos), con los instrumentos contables conocidos y con los que dictan los principios contables. Pero esto, no es suficiente, va a llegar el momento en que la actual contabilidad agote todo su repertorio contable para registrar los activos intangibles y estamos cerca.

¿Qué debemos hacer?

He dicho que el análisis contable actual es poco adecuado (no digo inadecuado), para valorar los intangibles y más bien se debe apoyar o ayudar de otros instrumentos - herramientas que nos permitan acercarnos a su valoración y/o medición.

No olvidemos, que la característica más importante del activo intangible es que crece de valor en el tiempo, es decir en el futuro, se acrecienta con el paso de los años.

Entonces, debemos pensar en el futuro, cuando hablemos de intangibles. La Contabilidad entonces debe ser teleológica, es decir, debe mirar distante $y$ a futuro. Es por 
eso, que ya se habla del nuevo criterio contable basado en la información prospectiva. $\mathrm{Al}$ respecto mi colega Elsa Choy Zevallos nos señala que "Hoy las decisiones se toman sobre la base de información del presente y del futuro; no es suficiente la información contenida en los estados contables históricos, elaborada y publicada con relación a lo ocurrido en el pasado. Para los agentes económicos la información particularmente importante es la que les permite analizar y considerar las posibilidades de eventos futuros que deberán afrontar la empresa”.

\section{INFORMACIÓN FINANCIERA PROSPECTIVA}

La Norma Internacional sobre Compromiso de Aseguramiento (NICA) 3400, señala lo siguiente:

Significa información financiera basada en estimaciones sobre eventos que pueden ocurrir en el futuro y posibles acciones de una organización. Es altamente subjetiva en naturaleza y su preparación requiere el ejercicio de considerable criterio. La información financiera prospectiva puede tomar la forma de pronóstico, una proyección o una combinación de ambos, por ejemplo, un pronóstico de un año más una proyección de cinco años.

Mi amigo, Alfonso Chung Pinzas, profesor en la Facultad de Ingeniería Industrial de la UNMSM, al respecto señala que el estudio del futuro mediante la prospectiva es un tema de actualidad en la cual muchas organizaciones se preparan para afrontar el futuro que viene o para construir el suyo propio.

Lo que se precisa es una orientación de hacia dónde va la organización en el largo plazo, que futuro se desea construir, para evitar así el libre albedrío en la elaboración de este documento, lo cual muchas veces puede generar en visiones incorrectas, en cambios de rumbo bruscos, en otras palabras, hacia un futuro incierto.

\section{DOS CONCEPCIONES DEL FUTURO}

Tenemos varias formas de concebir el futuro para una organización, sin embargo, para este trabajo de investigación, tenemos 2 , que a nuestro juicio, son los que mejor se adecuan al ámbito contable:
a. Forecasting ó Forecast
b. Foresight

\section{a. FORECASTING}

Fue una de las primeras herramientas para pronosticar el futuro, para inventarlo. Como sabemos el futuro no se puede predecir ni adivinar, pero si se puede inventar (hacerlo o llevarla a cabo).

Utiliza medias móviles, regresión simple y múltiple, alisados, entre otros.

Según su esquema de pensamiento, el futuro es único y está condicionado a la evolución estadística de las tendencias.

Recientemente, también se ha determinado y demostrado que el pasado y el presente muchas veces no tiene nada que ver con el futuro de una organización. Y es cierto, cuando cambian los paradigmas, todo vuelve a cero o a un inicio nuevo y así sucesivamente.

\section{b. FORESIGHT}

En cambio el Foresight (escuela anglosajona), constituyó un mejoramiento del Forescasting. Si bien conserva parte de sus principios, es decir, el análisis del futuro se basa en la evaluación de las tendencias, estas tendencias son analizadas en función de la opinión de expertos, por lo tanto la selección de dichos expertos es vital en este tipo de herramienta (la contabilidad lo hace a través de un equipo técnico de la fundación IFRS 
y otros equipos de trabajo de índole internacional).

El Foresight propone que el futuro si bien está condicionado por la evolución de las tendencias, las cuales se analizarán según la opinión de expertos, uno puede prepararse para este futuro tomando las medidas pertinentes para afrontarlo.

El Foresight, entre sus principales herramientas, tiene:

a. Técnica Delphi

b. Construcción de escenarios

c. Trabajos estructurales

d.Método de Escenarios / Análisis Morfológico

e. Análisis de impactos, entre otros

\section{UNA NUEVA CONCEPCIÓN DEL FUTURO: LA PROSPECTIVA ESTRATÉGICA}

La Prospectiva Estratégica, también conocida como la escuela francesa, la cual fue impulsada por el Dr. Michael Godet. En este caso se propone un rompimiento con el pensamiento de los métodos anteriores básicamente en dos aspectos:

El futuro no depende de la evolución de las tendencias sino de la relación que existe entre los factores del sistema, actores y objetivo. En efecto bajo esta perspectiva el futuro con los posibles escenarios futuribles se darán en función a cómo interactúan los agentes mencionados anteriormente, esto no deja de lado la importancia de las tendencias, pero indica que la relación entre los agentes tales como factores del sistema, actores y objetivo condicionará su comportamiento y por lo tanto el desarrollo de los eventos.

No existe un solo futuro sino varios escenarios futuros posibles, debido a la mencionado en el puesto anterior, se puede concluir que el comportamiento de los agentes que determinan el futuro del sistema puede ser variado, por lo tanto, no existe un único futuro sino varios futuros, lo que en prospectiva estratégica se conoce como "escenarios futuribles", lo cual representa una gran evolución respecto a las técnicas estudiadas y/o señaladas anteriormente.

En otras palabras, lo que se busca con la Prospectiva Estratégica no es pronosticar el futuro y estar resignado a él, o prepararse para afrontar ese futuro, al contrario la esencia de la Prospectiva Estratégica consiste en construir el futuro deseado lo que se conoce como escenario apuesta.

Trabajar con Prospectiva Estratégica implica también, el trabajo con expertos multidisciplinarios, convirtiendo lo cualitativo en cuantitativo, mediante sus herramientas de trabajo.

Entonces, la NIC-38, para un futuro cercano tendrá como uno de sus instrumentos de trabajo la Prospectiva Estratégica, que le permitirá no solo reconocer cuales son los activos intangibles, sino medirlos y posteriormente contabilizarlos.

Más aún, de los nuevos activos intangibles nacerán los nuevos tangibles; por ejemplo el Wall Street de Nueva York, se mueve por la fe, por creer en el futuro.

He allí, la enorme importancia que tendrá no solo la NIC-38 en el futuro, sino toda la contabilidad para enseñarnos la verdad de lo que ocurre en la empresa.

Creo entonces que ya tenemos un camino o derrotero a seguir, sobre todo para llegar a un consenso que tanto requerimos y necesitamos, para todos hablar un lenguaje común, concreto y asertivo.

A partir de aquí, creo y me reafirmo que la contabilidad se convertirá en la parte principal y más importante de todas las funciones de la empresa (como sabemos son: la función Administrativa, Financiera, Contable, Producción u Operaciones, Personal o RR.HH., Comercial o Marketing y Logística 
o Aprovisionamiento), y lo asumo que será para siempre, pues dará la información del todo, para una correcta toma de decisiones.

\section{CONCLUSIONES}

1. Los Activos Intangibles generan y dan valor a las cosas.

2. La actual Contabilidad, aún no puede registrar Contablemente los Activos Intangibles, salvo algunos cuantos.

3. En una empresa, existen más intangibles que tangibles, aún sea esta de producción o fabricación

4. Cada vez crece y/o aumenta el valor de las empresas por sus intangibles.

5. Existen Activos Intangibles que la Contabilidad a aceptado y otros Activos Intangibles que no los acepta como tal.

6. A medida que pasa el tiempo, los Intangibles crecen en valor, mientras que los productos se desvalorizan, se deterioran o simplemente se deprecian.

7. Existen nuevos instrumentos no financieros que sirven para medir Activos Intangibles.

8. Los Activos Intangibles se pueden medir a través de porcentaje, peso, volumen o masa y estos resultados deben ser colocados en los estados financieros de la empresa.

9. El actual análisis Contable es poco adecuado para valorizar los Otros Activos Intangibles (tales como la lealtad, la sinergia, la satisfacción del cliente, know how, reputación, clima laboral, liderazgo, entre otros)

10. Ya sé a empezado a darle la verdadera importancia que tienen los intangibles en la empresa.

\section{REFERENCIAS BIBLIOGRÁFICAS}

1. NIC-38 Activos Intangibles. Equipo Técnico de la Fundación IFRS. 1 de enero del 2012

2. ¿Qué es un Activo Intangible?. Cañibano García - Ayuso y Sánchez.1959

3. Activos Intangibles. Tania Delgado Martínez 2009

4. Asignatura: Psicología Organizacional. Facultad de Ciencias Contables UNMSMMedición de los Intangibles (separata). Arturo Valdivia Ramos. 2010

5. Asignatura: Administración General II. Facultad de Ciencias Contables UNMSM. El futuro de los Intangibles (separata). Arturo Valdivia Ramos. 2011

6. Revista Quipukamayoc Julio-Diciembre 2011, Volumen 19, $\mathrm{N}^{\circ}$ 36, UNMSM, Lima, Perú. Nuevo criterio Contable basado en la información prospectiva. Elsa Esther Choy Zevallos

7. Revista de la Facultad de Ingeniería Industrial - UNMSM Industrial Data - Revista de Investigación. Volumen 12, N², Julio-Diciembre 2009, Perú. Prospectiva Estratégica: Mas allá del plan Estratégico. Alfonso Chung Pinzas 